\title{
LIMITS TO VLBI RESOLUTION DUE TO SOLAR CORONAL SCATTERING
}

\author{
Stanislaw Gorgolewski \\ Torun Radio Astronomy Observatory \\ Nicolaus Copernicus University \\ Torun, Poland
}

\begin{abstract}
Electron irregularities in the solar corona scatter the radiation from radio sources. During more than 30 years of coronal electron irregularity investigations, a very complex temporal and spatial behavior of the solar corona have been observed. In particular, the scattering dependence on distance from the Sun varies with both the phase of the cycle and the different solar maxima. The influence of scattering on the highest resolution observations in radio astronomy, i.e., those made with VLBI, is not negligible. It should be taken into account in planning of both the global VLBI as well as the future orbital VLBI stations such as the QUASAT and RADIOASTRON missions. The "normal" scattering dependence on the distance from the Sun is close to the inverse square law, but substantial deviations from this dependence have been found.
\end{abstract}

\section{INTRODUCTION}

The irregular structure of the solar coronal electron density was discovered in the early fifties, during the search for refraction during the occultation of Tau A by the solar corona. No refraction was found, but scattering, which causes an apparent increase of the observed radio source diameter, was detected. Interferometers of about 100 wavelength spacings were used in these measurements in the meter wavelength range. More recently, small diameter sources have been observed with proportionally longer baselines at decimeter waves. With the discovery of interplanetary scintillations, finer structure in radio sources could be observed; this led the way to the discovery of the pulsars. Since scattering is proportional to wavelength squared, long wavelength observations were preferred for higher sensitivity.

Vast observing materials have become available during the last three decades. Anisotropic scattering provided evidence for the existence of radial magnetic fields in the outer solar corona. With the achievement of high resolution by VLBI, solar coronal scattering gains new importance. It may soon become the chief limiting factor in highest resolution observations at long decimetric waves using the longest ground-based baselines, as well as space VLBI baselines. 


\section{RESULTS}

Scattering without refraction was found to be the main effect of the passage of radio waves through the solar corona at distances up to several tens of solar radii. These results refer to medium baseline interferometers at meter waves during solar maxima. Interplanetary scintillations can be observed on very small diameter sources at distances comparable to the astronomical unit, i.e., about 200 solar radii. The most surprising result was the discovery made in 1969 by both radio source occultations and interplanetary scintillations that solar coronal scattering did not follow the presumed correlation with the solar activity cycle. It was found in 1970 that the scattering was indeed stronger than ever before observed, i.e., in 1959. It existed only at rather small distances from the Sun. At the distance of 10 solar radii from the Sun, the scattering did not differ from that during the solar minimum. Another surprise came in 1979 , when the scattering dependence on distance from the Sun followed an inverse 1.24 power law, the flattest dependence yet observed. One can thus see that the solar corona does not behave predictably. We must monitor the ever changing conditions of the Sun and its influence on the solar corona if we want to avoid surprises.

\section{DISCUSSION}

The simple idea of an 11-year solar cycle does not fully account for all observed scattering events as related to both the distance and phase of the solar cycle. One should take the 22-year period of solar activity as the full cycle. This total period of solar magnetic activity contains the full cycle of magnetic field evolution. During solar field reversal, the magnetic field lines are wrapped around the corona. Normally, the radial field lines permit the solar wind to flow from the Sun, but during the time of field reversal, the magnetic lines inhibit the flow from the solar regions. Should this pattern of events be the long-term solar behavior, the next most favorable observing period for highest resolution VLBI should come near the next solar maximum, i.e., near 1991. This period is near the time of the expected QUASAT and RADIOASTRON missions. The following solar maximum near the year 2002 may well be the time of the extended solar coronal irregularities - a time not very suitable for highest resolution VLBI.

I thank the I.A.U. for subsistence and a travel grant in the U.S.A. Other travel expenses as well as financial support for research were taken care of by the "Resortowy Program Badan Rozwojowych": RPBR NR. RR. I. 11/2.

\section{BIBLIOGRAPHY}

Erickson, W. C. 1964, Ap. J., 139, 1290.

Gorgolewski, S., and Hewish, A. 1960, The Observatory, 80, 99.

Gorgolewski, S., and Krygier, B. J. 1977, Interdiscipl. Cycle Res. 8(3-4), 189.

Hewish, A. 1958, M. N. R. A. S., 118, 534.

Hewish, A., Dennison, P. A., and Pilkington, J. D. H. 1966, Nature, 209, 1188.

Hewish, A., Wyndham, J. D. 1963, M. N. R. A. S., 126, 469.

Little, L. T., Hewish, A. 1966, M. N. R. A. S., 134, 221.

Okoye, S. E., and Hewish, A. 1967, M. N. R. A. S., 137, 287.

Slee, O. B. 1961, M. N. R. A. S., 123, 223.

Vitkevich, V. V. 1960, A. J. U.S.R.R., 37, 32. 